\title{
Letter to the editor: Olynk and Wolf (2007) stands correct as published as a method to estimate the net present value of different sexed semen artificial insemination strategies in dairy heifers
}

\author{
N. J. Olynk ${ }^{\star 1}$ and C. A. Wolf† \\ *Department of Agricultural Economics, Purdue University, West Lafayette, IN 47907 \\ †Department of Agricultural Economics, Michigan State University, East Lansing 48825
}

The comment provided by Joezy-Shekalgorabi and Shadparvar (2012) in a Letter to the Editor in this issue displays a clear misunderstanding of our analysis and our model as defined. We will address the comment for clarity and interpretation purposes and provide a simple example of why the authors' assertion of bias in Olynk and Wolf (2007) is unsupported by their own equation. In fact, their equation actually yields a lower net present value (NPV) than that in our original paper.

The fact that a heifer remains under consideration in time period $t$ or $T$ (in the model as defined Olynk and Wolf, 2007) means, by definition, that she is open (has not yet achieved the $90 \%$ probability of pregnancy, as defined in our paper) and will, therefore, be bred. Our probabilistic treatment of the situation does not seek to estimate the value (or cost) of any single heifer being bred. This is clear in the original publication. Rather, we calculated the cumulative probability of pregnancy, the probability of obtaining a heifer versus bull calf, and so on, to value the breeding strategy. Because the heifer is open - and being bred-the insemination cost will be incurred with certainty each period that the heifer is bred. The suggestion by Joezy-Shekalgorabi and Shadparvar (2012) that insemination cost is probabilistic is incorrect. They suggest that the expected insemination cost can be no greater than the total cost of a single insemination, regardless of how many times the animal actually needs to be bred to achieve a $90 \%$ cumulative probability of pregnancy. Again, the fact that heifer is still in $t$ or $T$ means that she is open. It is given by the setup of the problem that no probability is assigned to whether or not the heifer enters the next breeding period; if she has not achieved the $90 \%$ cumulative probability of pregnancy, she is open and bred with $100 \%$ certainty. The heifer is either open

Received March 9, 2012.

Accepted August 16, 2012.

${ }^{1}$ Corresponding author: nolynk@purdue.edu or pregnant. If the heifer is open, and bred, the full insemination cost is incurred.

The crux of their argument is as follows:

\begin{abstract}
"Olynk and Wolf (2007) obtained the number of service per conception for achieving a minimum cumulative pregnancy rate $\left(\boldsymbol{P} \boldsymbol{R}_{\text {cum }}\right)$ of $90 \%$. The services per conception necessary to achieve this assumption was greater for strategies based on pure or mixed sexed semen AI compared with that based on conventional semen. Thus, it could be concluded that the double counting of insemination costs would have more effect when predicting NPV of sexed semen breeding strategies."
\end{abstract}

As is clear from the discussion above, Joezy-Shekalgorabi and Shadparvar (2012) are incorrect in their adjustment of our insemination costs. Further, the conception rate in a given service with sexed semen is less than that with conventional semen, as is cited and explained in our original paper. Therefore, when a strategy incorporating sexed semen (whether a mixed strategy or just sexed semen alone), the number of services required to obtain the $90 \%$ cumulative pregnancy rate (and be considered bred) is higher than if just conventional semen was used. This is indeed correct and costs are incurred. There is a "cost" in terms of lower conception rate of using sexed semen. That cost correctly leads to a larger number of services required to obtain a $90 \%$ conception rate. Our analysis correctly highlighted the trade-off between lower conception rate and higher calf value with sexed semen. The intention was to demonstrate the trade-offs and breakevens, rather than assign a value.

We should also point out that the "corrected" equation in Joezy-Shekalgorabi and Shadparvar (2012) multiplies the value of the calf by the probability of being open in that period. This is incorrect because the value of the calf in period $t$ is already multiplied by the conception rate in that period. This misunderstanding further highlights that the authors did not understand the model or its implications. 
With the value of the calf and insemination cost multiplied by a value $<1.0$ (the probability of conception in that period), Joezy-Shekalgorabi and Shadparvar (2012) derive a NPV for sexed semen that is less than the value derived using the correct formula in Olynk and Wolf (2007). To understand this, consider a 3-period model with the modification of Joezy-Shekalgorabi and Shadparvar (2012) compared with the model defined by us, and compare the resulting NPV. Assuming that all parameter values are the same, after cancelling out like terms, Joezy-Shekalgorabi and Shadparvar (2012) are left with $\left(1-P R_{\text {cum }}\right) \times 2 \times(\mathrm{CR} \times \mathrm{VAC}-\mathrm{IC})$, whereas we had $2 \times(\mathrm{CR} \times \mathrm{VAC}-\mathrm{IC})$, where $P R_{\text {cum }}$ $=$ cumulative pregnancy rate, $\mathrm{CR}=$ conception rate, $\mathrm{VAC}=$ value of average calf, and $\mathrm{IC}=$ insemination cost. Denoting $2 \times(\mathrm{CR} \times \mathrm{VAC}-\mathrm{IC})=\beta$, the above comparison can be simplified to $\left(1-P R_{\text {cum }}\right) \times \beta$ in Joezy-Shekalgorabi and Shadparvar (2012) and to $\beta$ in Olynk and Wolf (2007). Note that $\beta$ is a positive number for all relevant and reasonable calf values and inseminations costs, and $\left(1-P R_{\text {cum }}\right)$ is a number $<1.0$. Because Joezy-Shekalgorabi and Shadparvar (2012) are multiplying $\beta$ by a number $<1.0$ or $\left(1-P R_{\text {cum }}\right)$, their NPV is smaller than that in our original paper. Thus, not only is the proposed "fix" from Joezy-Shekalgorabi and Shadparvar (2012) incorrect and inconsistent conceptually, but it would result in a smaller NPV for sexed semen. If we were "biased" downward with respect to sexed semen NPV, then Joezy-Shekalgorabi and Shadparvar (2012) are even more biased using the equation that they propose.

Because Joezy-Shekalgorabi and Shadparvar (2012) specifically state "Definition of all the parameters are the same as those defined by Olynk and Wolf (2007)," a different interpretation of our notation and model is inappropriate. Thus, their proposed calculation is incorrect with respect to both insemination cost and calf value.

\section{REFERENCES}

Joezy-Shekalgorabi, S., and A. A. Shadparvar. 2012. Letter to the editor: Underestimation of the net present value of different sexed semen artificial insemination strategies in dairy heifers: A comment on Olynk and Wolf (2007). J. Dairy Sci. 95:6185.

Olynk, N. J., and C. A. Wolf. 2007. Expected net present value of pure and mixed sexed semen artificial insemination strategies in dairy heifers. J. Dairy Sci. 90:2569-2576. 